\title{
HST/NICMOS observations of a proto-brown dwarf candidate
}

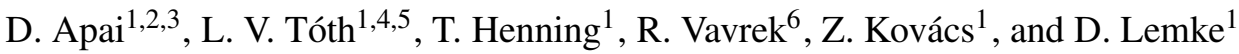 \\ ${ }^{1}$ Max Planck Institute for Astronomy, Königstuhl 17, 69117 Heidelberg, Germany \\ e-mail: apai@as.arizona.edu \\ 2 Steward Observatory, The University of Arizona, 933 N. Cherry Avenue, Tucson, AZ 85721, USA \\ ${ }^{3}$ NASA Astrobiology Institute \\ 4 Department of Astronomy of the Loránd Eötvös University, Pázmány Péter sétány 1, 1117 Budapest, Hungary \\ 5 Konkoly Observatory of the Hungarian Academy of Sciences, PO Box 67, 1525 Budapest, Hungary \\ ${ }^{6}$ Astrophysics Missions Division, ESTEC, Keplerlann 1, Norwijk 2201, The Netherlands
}

Received 6 December 2004 / Accepted 18 February 2005

\begin{abstract}
We present deep HST/NICMOS observations peering through the outflow cavity of the protostellar candidate IRAS 04381+2540 in the Taurus Molecular Cloud-1. A young stellar object as central source, a jet and a very faint and close $\left(0.6^{\prime \prime}\right)$ companion are identified. The primary and the companion have similar colours, consistent with strong reddening. We argue that the companion is neither a shock-excited knot nor a background star. The colour/magnitude information predicts a substellar upper mass limit for the companion, but the final confirmation will require spectroscopic information. Because of its geometry, young age and its rare low-mass companion, this system is likely to provide a unique insight into the formation of brown dwarfs.
\end{abstract}

Key words. binaries: close - planetary systems: protoplanetary disks - stars: formation - stars: low-mass, brown dwarfs stars: pre-main sequence

\section{Introduction}

The debate on the origin of brown dwarfs has preceded their discovery (Rebolo et al. 1995): their small masses poses serious difficulty to a stellar-like formation scenario through the collapse of a molecular cloud core. Now, with hundreds of known brown dwarfs, the debate is still not settled: the most widely discussed formation scenarios include gravitational collapse in highly turbulent cloud cores (e.g. Padoan \& Nordlund 2004), early ejection from unstable multiple systems (Sterzik \& Durisen 1998; Reipurth \& Clarke 2001; Umbreit et al. 2004) and photoevaporation of protostars (Whitworth \& Zinnecker 2004). Only few traces of the formation process survive the early evolution of these objects. Current studies focused on disk indicators, signs of accretion, binarity and velocity dispersion. We learned that brown dwarfs are often surrounded by warm circumstellar material (e.g. Jayawardhana et al. 2003), with masses of $\sim M_{\text {Jup }}$ (Klein et al. 2003) which they maintain over timescales similar to stars (Sterzik et al. 2004), and in one case it could be shown that the dust forms a disk (Pascucci et al. 2003). Because gravitational ejection leaves sufficient material in the inner disk (Bate et al. 2002) to produce infrared excess emission and accretion signs, the ejection embryo hypothesis remains a possible formation scenario. The masses derived from millimetre-wavelength observations
(Klein et al. 2003) are too uncertain to constrain possible disk truncation and therefore cannot exclude the ejection scenario.

Direct observations of the youngest phases of substellar objects, termed as proto-brown dwarfs, will be crucial in evaluating the formation scenarios. Recent imaging by Haisch et al. (2004) and Duchêne et al. (2004) identified faint, possibly substellar companion candidates to protostellar systems, but some of them might be background stars. To ensure the very young age of the proto-brown dwarfs, they should be observed within the massive protostellar envelope, possible only in exceptional cases.

We present here observations of the first such system, IRAS $04381+2540$, located in TMC-1 in the Taurus starforming region with the discovery of a proto-brown dwarf candidate. Based on its spectral energy distribution and bolometric temperature, IRAS $04381+2540$ has been classified as a Class I object (Hogerheijde \& Sandell 2000). However, estimates of the stellar mass $\left(M=0.3 \pm 0.1 M_{\odot}\right.$, Brown \& Chandler 1999) show that it is comparable to the envelope mass $\left(M=0.18 M_{\odot}\right.$, Young et al. 2003) indicating that the object is still in its main accretion phase (Motte \& André 2001). IRAS 04381+2540 drives a molecular outflow (Brown \& Chandler 1999), with an inclination of $i \approx 50^{\circ}$ (the northern part tilted toward the observer, Chandler et al. 1996). Through the evacuated outflow cone we see into the dense molecular cloud core in which 
Table 1. Log of the infrared observations and results of the NICMOS photometry. The estimated photometric error is less than $10 \%$ for all bands. In the $F 212 N$ band only an upper limit can be reliably established due to the low contrast between the object and the background nebulosity. Here, the use of a smaller aperture reduces the contamination from the nebulosity and places a stricter upper limit. The $F 160 \mathrm{~W}$ filter also includes [Fe II] line emission at $1.644 \mu \mathrm{m}$.

\begin{tabular}{|c|c|c|c|c|c|c|c|c|c|}
\hline \multirow[t]{2}{*}{ Instrument } & \multirow[t]{2}{*}{ Filter } & \multirow{2}{*}{$\begin{array}{c}\text { Date } \\
\text { DD/MM/YY }\end{array}$} & \multirow{2}{*}{$\begin{array}{c}\text { Field of } \\
\text { view }\end{array}$} & \multirow{2}{*}{$\begin{array}{c}\text { Exp. time } \\
{[\mathrm{s}]}\end{array}$} & \multicolumn{2}{|c|}{ Flux of NIR A } & \multicolumn{2}{|c|}{ Flux of NIR B } & \multirow[t]{2}{*}{ Note } \\
\hline & & & & & {$[\mu \mathrm{Jy}]$} & [mag] & {$[\mu \mathrm{Jy}]$} & [mag] & \\
\hline HST/NICMOS & $F 160 W$ & $20 / 12 / 97$ & $20^{\prime} \times 19^{\prime}$ & 1280 & 511 & 15.8 & 38 & 18.6 & $1.4-1.8 \mu \mathrm{m}$ \\
\hline HST/NICMOS & $F 205 W$ & 07/01/98 & $19^{\prime} \times 19^{\prime}$ & 256 & 6295 & 12.6 & 363 & 15.7 & $1.75-2.35 \mu \mathrm{m}$ \\
\hline HST/NICMOS & $F 212 N$ & 07/01/98 & $19^{\prime} \times 19^{\prime}$ & 160 & 7711 & 12.3 & $<1070$ & $>14.5$ & $2.121 \mu \mathrm{m}, \mathrm{H}_{2}$ \\
\hline HST/NICMOS & $F 212 N$ & 07/01/98 & $19^{\prime} \times 19^{\prime}$ & 160 & & & $\leq 351$ & & $0.13^{\prime \prime}$ aper. \\
\hline
\end{tabular}

IRAS $04381+2540$ is embedded, making it an exceptional target for studies of very early stellar evolution.

\section{Observations and data reduction}

We used archival HST/NICMOS observations to study IRAS $04381+2540$ at small spatial scales. The main parameters of the observations are summarised in Table 1.

The data have been successfully pipeline-processed and required only minor additional reduction work, which was carried out by simple IDL scripts. The images have been bad pixel filtered and the central columns bias-corrected in the standard fashion. The final mosaic has been composed by crosscorrelating the individual pointings. In order to enhance the fainter details around the central point source we subtracted synthetic point spread functions (PSF) calculated by using the Tiny Tim 6.1 (Krist \& Hook 1997). The scaling factors have been obtained by comparing the integrated counts in 3-pixelradii apertures placed on the simulated and observed peaks.

Aperture photometry has been performed on all images in an identical way. We used the IDL adaptation of the DAOPHOT routine to integrate the counts in 6-pixel-radius apertures centered on the central point source. In order to obtain reliable photometry also for the fainter, secondary point source we carried out PSF photometry by subtracting simulated PSFs. By minimizing the subtraction residuals we measured the point source's brightness without significant contribution from the surrounding nebulosity. In the case of the $F 212 N$ filter, due to the faintness of the secondary point source compared to the nebula, we could only estimate upper limits from the aperture photometry. Here, we also applied a second, small $3 \times 3$ pixel aperture to suppress the contamination from the nebula and thus obtained a stricter upper limit.

\section{Results}

As shown in Fig. 1 the NICMOS image reveals a detailed view of the reflection nebulosity including two point sources (IRAS 04381+2540 NIR A and IRAS 04381+2540 NIR B) and a long near-infrared jet at the heart of IRAS $04381+2540$. In the following, we will refer to the brighter, primary point source as NIR A and to the fainter, secondary one as NIR B. NIR A is located in the apparent center of symmetry of the reflection nebula with the position of RA: $04^{\mathrm{h}} 41^{\mathrm{m}} 12^{\prime \prime} .65$ Dec: $+25^{\circ} 46^{\prime} 35^{\prime \prime} \cdot 93$ (J2000). This position is accurate within

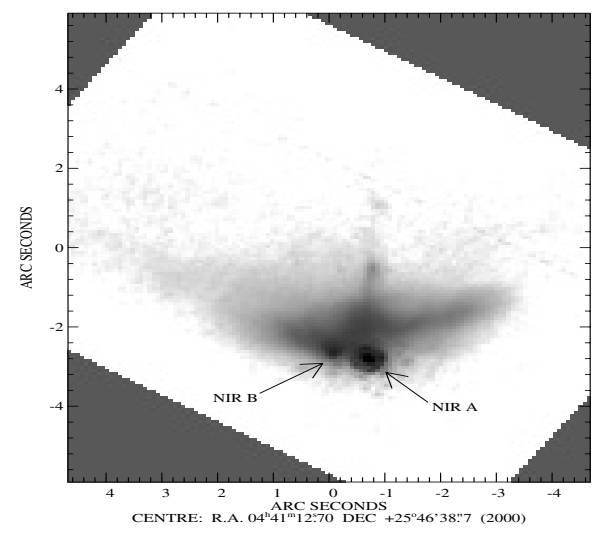

Fig. 1. High-resolution near-infrared view of IRAS $04381+2540$ by HST/NICMOS in the broadband $F 160 W$ filter. This image reveals a binary young stellar object (NIR A+B) and a collimated jet.

the spacecraft's pointing accuracy, typically better than $0{ }^{\prime} 5$. All NICMOS images show NIR A as a point source at a resolution of $0 !^{\prime} 15$. The secondary source NIR B is located at 0 '59 east and 0 ' 15 north of NIR A. The source is unresolved in all three NICMOS images, while in the $F 160 \mathrm{~W}$ image a possible marginal, faint extension is seen at the brightness level of the surrounding nebula, possibly a faint scattered-light envelope of NIR B. The photometry results are summarized in Table 1 . The deepest $F 160 \mathrm{~W}$ image also reveals the presence of a $3^{\prime \prime} 3$ long, narrow line of intense emission, which we identified as a highly collimated jet. While the northern end of the jet vanishes in the background noise about $5^{\prime \prime}$ from NIR A, its southern end is overshined by the reflection nebula at about $1.2^{\prime \prime}$ from NIR A. The jet's direction points to NIR A identifying its driving source.

\section{Discussion}

The detection of the collimated jet proves that NIR A, its driving source, is still in the early, accreting phase of its evolution. By assuming an inclination of $i=50^{\circ}$ for the jet its visible length is $\sim 1000 \mathrm{AU}$. As often observed, the jet in IRAS $04381+2540$ is accompanied by a molecular outflow (Chandler et al. 1996). The present and former studies suggest the following picture: the young stellar object NIR A at a transition stage in the Class 0/Class I boundary - is located close to the side of the dense molecular cloud core facing the observer. The low-mass source NIR A drives a collimated 


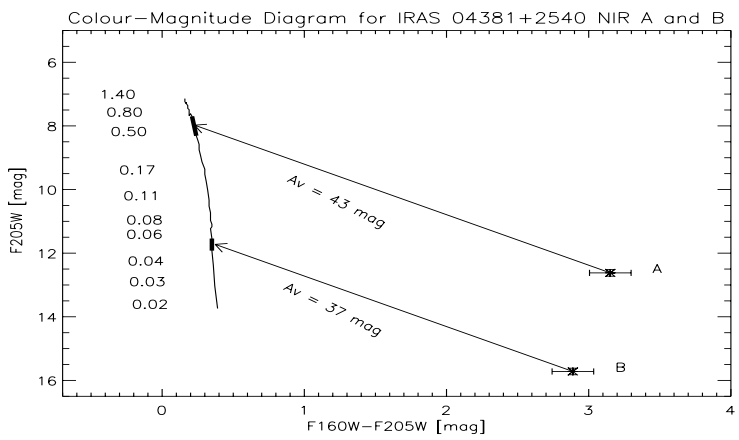

Fig. 2. Colour-magnitude diagram based on HST/NICMOS photometry for the IRAS $04381+2540$ NIR A and B sources. The 1 Myr-old isochrone from Baraffe et al. (1998) is overplotted. The numbers indicate the masses in solar units. The interstellar reddening vectors for $A_{\mathrm{V}}=43 \mathrm{mag}$ and $37 \mathrm{mag}$ are also plotted. Thick lines mark the approximate mass range as constrained by the photometric errors.

jet and harbors a circumstellar accretion disk perpendicular to the jet's direction. The jet-powered outflow clears out a lowdensity cavity, whose wall becomes bright by scattering the light of NIR A. The southern jet is inclined away from the observer and, by penetrating into the denser regions of the cloud, becomes strongly extincted and apparently fainter. The northern beam of the jet is inclined toward the observer and is visible inside the outflow cavity. The age of the system can be estimated on the basis of the $\sim 10^{5}$ yr typical lifetime of protostellar objects, consistent e.g. with the chemical evolution studies of the deuterium fractionation (Saito et al. 2002) in IRAS $04381+2540$.

\subsection{The nature of the point sources}

The near-infrared colour information allows preliminary conclusions on the nature of NIR A. When plotted in a colourmagnitude diagram (see Fig. 2) the photometry of NIR A is consistent with that of a highly reddened $\left(A_{\mathrm{V}} \approx 43 \mathrm{mag}\right)$ young stellar object. The mass estimate of $\sim 0.6 M_{\odot}$ based on the comparison of the near-infrared photometry to the $1 \mathrm{Myr}$ isochrones of Baraffe et al. (1998) and is consistent with the observed bolometric luminosity $\left(0.7 L_{\odot}\right.$, Chandler et al. 1998). A similar mass estimate was derived from gas kinematics $\left(0.3 \pm 0.1 M_{\odot}\right.$, Brown \& Chandler 1999). The case of NIR B is more compelling; as NIR B does not have any evident link to its environment suggesting it to be a companion of NIR A, it could also be an unrelated background/foreground star, or an excited knot in the cavity wall. These three possibilities are briefly discussed below.

(1) Foreground/background source: the high reddening of NIR $\mathrm{B}\left(A_{\mathrm{V}} \approx 37 \mathrm{mag}\right)$ excludes the possibility of an unrelated foreground star. The comparison (Balázs et al. 2004) of the Wainscoat et al. (1992) model to the 2MASS Point Source Catalogue predicts $A_{\mathrm{V}} \approx 16$ mag extinction towards our source and the probability of a chance background star appearing within $\sim 1.5$ square arcsecond to be $\approx 10^{-4}$. The additional $A_{\mathrm{V}} \gtrsim 20$ mag extinction observed towards IRAS $04381+2540$ makes such a coincidence even less likely.
(2) A shock-excited knot associated to the jet: although several such examples are known, e.g., in L1551 NE (e.g. Reipurth et al. 2000), the source NIR B differs from these objects in three important aspects: it is visible and unresolved in the broad-band images; and its brightness in the $F 212 N$ filter is dominated by continuum emission (i.e. no shocked $\mathrm{H}_{2}$ emission is visible).

(3) Companion to NIR A: NIR B has near-infrared colours very similar to NIR A; a comparison to the 1 Myr-old substellar models of Baraffe et al. (1998) suggests a reddening almost identical to NIR A (see Fig. 2). In addition to the matching colours, the very proximity of NIR A and B further reinforces their physical link. The alternative scenarios being very improbable, in the following we tentatively accept NIR B to be a point source related to NIR A.

\subsection{Probable substellar mass for NIR B}

The comparison of NIR B brightness and colour (see Fig. 2) argues for a substellar mass of $M_{B} \simeq 50 \pm 4 M_{\mathrm{Jup}}$. The error bar represents the photometric error, but in the following we discuss several other factors which influence this estimate.

(1) Isochrones: our object is likely to be younger than the youngest available isochrones dating 1 Myr. The yet unknown initial conditions of brown dwarf formation probably introduce significant uncertainities even for the 1 Myr tracks (Baraffe et al. 2002). Comparing the luminosity and effective temperature in the 40-60 $M_{\text {Jup }}$ range to the 1 Myr-old models by Burrows et al. (1997), we find that the latter models associate 3-5 $M_{\text {Jup }}$ smaller masses to the given luminosities, suggesting an even smaller mass for NIR B. Although no isochrones of the appropriate age are yet available, using overaged isochrones lead to the overestimation of the mass and therefore enables us to set an upper mass limit for NIR B of $\simeq 50 M_{\text {Jup }}$.

(2) Reddening: the reddening law plays a crucial role when interpreting objects as heavily reddened as NIR B. In Fig. 2 we used the reddening law by Mathis (1990) and now we explore how the estimate varies with the reddening law's slope. A study of the reddening toward the Taurus region by Whittet et al. (2001) showed that in the direction of dense clouds $R_{V}=3.5-4.0$ and might reach 4.5 for the densest regions of the cloud. Disentangling the contribution to NIR B's reddening by the different dust types is not possible. By making the worst-case estimate (assuming that all the extinction is caused by dust with $R_{V}$ as high as 4.5 ) would modify NIR B's upper mass limit to $\simeq 90 M_{\text {Jup }}$, i.e. slightly above the substellar limit. We conclude, that although the reddening law influences the mass estimates, it is very unlikely to shift the upper mass limit into the stellar regime.

(3) Anisotropic emission: the emission of embedded stars is often influenced by the circumstellar environment via obscuration and light scattering, exemplified by edge-on disks (see, e.g. Padgett et al. 1999). In these cases both the colour and the magnitude of the objects are altering mass estimates on the basis of theoretical isochrones. Circumstellar material obscuring more than $66 \%$ of NIR B's emission could apparently shift the luminosity of a very low mass star to substellar luminosities. Alternatively, NIR B's emission might originate partly 
by its envelope, scattering the light of NIR A; then NIR B's mass might be even smaller than estimated. Entirely excluding these possibilities is impossible without detailed spectroscopic and polarimetric follow-up observations but we argue that such a strong scattered-light nebula would likely be resolvable by our observations. While a study by Padgett et al. (1999) shows 7 such nebulae around edge-on disks to have typical sizes of $\simeq 800 \mathrm{AU}, \mathrm{NIR}$ B is an unresolved point source at our $\sim 20 \mathrm{AU}$ resolution. Thus, should NIR B be a reflection nebula, its point source nature would be rather exceptional.

\subsection{NIR B as a brown dwarf in formation}

The concept of NIR B being a substellar companion of NIR A opens up exciting possibilities. Although several stars with brown dwarf companions are known, this object is about an order of magnitude younger than the previously known youngest brown dwarf companion to the T Tauri star GG Tau Bb (White et al. 1999). The presence of the jet confirms active accretion in the system and suggests that NIR B is a brown dwarf in formation. An important question is whether NIR B will be able to accrete sufficient mass to become a star during the future accretion processes. Observations of the $\mathrm{Br} \gamma$ line can determine the accretion rates of NIR A and NIR B, and show whether the current mass difference will increase (to produce a lowmass star with brown dwarf companion) or decrease (toward an equal-mass binary situation). Additional, longer wavelength high-resolution observations can identify whether NIR B is embedded in the disk of NIR A or harbors its own accretion disk, an important clue on the formation history of the system. Should NIR B be in the disk, it is likely to gravitationally interact with the disk and its mass is probably sufficient to induce observable structures, similar to the ones predicted for massive planets embedded in Solar-type stars' disks (e.g. Bryden et al. 2000; D'Angelo et al. 2003) allowing the observational test of these models.

\section{Conclusions}

Based on our HST/NICMOS infrared imaging the main conclusions of this work are the following:

(i) The central source of the IRAS $04381+2540$ system is identified as a young, low-mass binary stellar object.

(ii) The accreting primary component drives a collimated jet.

(iii) The secondary component is probably the youngest known brown dwarf so far, providing a unique opportunity to study the formation of substellar objects.

Acknowledgements. The HST data have been obtained in the frame of the programmes 7325 and 7413 (PIs: L. Hartmann and S. Terebey). We are grateful to I. Baraffe for providing the isochrone data for the HST filter set. Valuable comments by I. Pascucci, W. Brandner, G. Schneider, L. Close, J. Muzerolle, R. Mundt and M. Goto, and technical help by $\mathrm{T}$. Khazadyan are acknowledged. The prompt and constructive review by our referee, G. Duchêne strengthened the science case and improved its presentation. This material is partly based upon work supported by NASA through the NASA Astrobiology Institute under Cooperative Agreement No. CAN-02-OSS-02. This research was partly supported by the OTKA grant T- 043774 .

\section{References}

Balázs, L. G., Ábrahám, P., Kun, M., Kelemen, J., \& Tóth, L. V. 2004, A\&A, 425, 133

Baraffe, I., Chabrier, G., Allard, F., \& Hauschildt, P. H. 1998, A\&A, 337, 403

Baraffe, I., Chabrier, G., Allard, F., \& Hauschildt, P. H. 2002, A\&A, 382,563

Bate, M. R., Bonnell, I. A., \& Bromm, V. 2002, MNRAS, 332, L65

Brown, D. W., \& Chandler, C. J. 1999, MNRAS, 303, 855

Bryden, G., Różyczka, M., Lin, D. N. C., \& Bodenheimer, P. 2000, ApJ, 540, 1091

Burrows, A., Marley, M., Hubbard, W. B., et al. 1997, ApJ, 491, 856

Chandler, C. J., Barsony, M., \& Moore, T. J. T. 1998, MNRAS, 299, 789

Chandler, C. J., Terebey, S., Barsony, M., Moore, T. J. T., \& Gautier, T. N. 1996, ApJ, 471, 308

D'Angelo, G., Kley, W., \& Henning, T. 2003, ApJ, 586, 540

Duchêne, G., Bouvier, J., Bontemps, S., André, P., \& Motte, F. 2004, A\&A, 427, 651

Haisch, K. E., Greene, T. P., Barsony, M., \& Stahler, S. W. 2004, AJ, 127,1747

Hogerheijde, M. R., \& Sandell, G. 2000, ApJ, 534, 880

Jayawardhana, R., Ardila, D. R., Stelzer, B., \& Haisch, K. E. 2003, AJ, 126, 1515

Klein, R., Apai, D., Pascucci, I., Henning, T., \& Waters, L. B. F. M. 2003, ApJ, 593, L57

Krist, J. E., \& Hook, R. N. 1997, in The 1997 HST Calibration Workshop with a New Generation of Instruments, 192

Mathis, J. S. 1990, ARA\&A, 28, 37

Motte, F., \& André, P. 2001, A\&A, 365, 440

Padgett, D. L., Brandner, W., Stapelfeldt, K. R., et al. 1999, AJ, 117, 1490

Padoan, P., \& Nordlund, A.. 2004, ApJ, 617, 559

Pascucci, I., Apai, D., Henning, T., \& Dullemond, C. P. 2003, ApJ, 590, L111

Rebolo, R., Zapatero-Osorio, M. R., \& Martin, E. L. 1995, Nature, 377,129

Reipurth, B., \& Clarke, C. 2001, AJ, 122, 432

Reipurth, B., Yu, K., Heathcote, S., Bally, J., \& Rodríguez, L. F. 2000, AJ, 120, 1449

Saito, S., Aikawa, Y., Herbst, E., et al. 2002, ApJ, 569, 836

Sterzik, M. F., \& Durisen, R. H. 1998, A\&A, 339, 95

Sterzik, M. F., Pascucci, I., Apai, D., van der Bliek, N., \& Dullemond, C. P. 2004, A\&A, 427, 245

Umbreit, S., Burkert, A., Henning, T., Mikkola, S., \& Spurzem, R. 2004, ApJ, in press

Wainscoat, R. J., Cohen, M., Volk, K., Walker, H. J., \& Schwartz, D. E. 1992, ApJS, 83, 111

White, R. J., Ghez, A. M., Reid, I. N., \& Schultz, G. 1999, ApJ, 520, 811

Whittet, D. C. B., Gerakines, P. A., Hough, J. H., \& Shenoy, S. S. 2001, ApJ, 547, 872

Whitworth, A. P., \& Zinnecker, H. 2004, A\&A, 427, 299

Young, C. H., Shirley, Y. L., Evans, N. J., \& Rawlings, J. M. C. 2003, ApJS, 145, 111 